\title{
EMBEDDINGS OF NONORIENTABLE SURFACES WITH TOTALLY REDUCIBLE FOCAL SET
}

\author{
by SHEILA CARTER and RIDVAN EZENTAS $\dagger$
}

(Received 21 May, 1992; revised 8 January, 1993)

1. Introduction. In an earlier paper [5] we introduced the idea of an immersion $f: M^{m} \rightarrow \mathbb{R}^{n}$ with totally reducible focal set. Such an immersion has the property that, for all $p \in M$, the focal set with base $p$ is a union of hyperplanes in the normal plane to $f(M)$ at $f(p)$. Trivially, this always holds if $n=m+1$ so we only consider $n>m+1$.

In [5] we showed that if $M^{2}$ is a compact surface then for all $n \geq 4$ there is a substantial immersion $f: M^{2} \rightarrow \mathbb{R}^{n}$ with totally reducible focal set. Further, if $M^{2}$ is orientable or is a Klein bottle or a Klein bottle with handles then $f: M^{2} \rightarrow \mathbb{R}^{n}$ can be taken to be an embedding. Here we show that if $M^{2}$ is a projective plane or a projective plane with handles then for all $n \geq 5$ there exists a substantial embedding $f: M^{2} \rightarrow \mathbb{R}^{n}$ with totally reducible focal set although, by arguments of $M$. Gromov and $E$. G. Rees, for $n=4$ such an embedding does not exist.

2. Notation and Definitions. Throughout this paper $M^{m}$ will denote a compact, connected, smooth $\left(C^{\infty}\right) m$-dimensional manifold without boundary. Let $f: M \rightarrow \mathbb{R}^{n}$ be a smooth immersion and, for $p \in M$, let $U$ be a neighbourhood of $p$ in $M$ such that $f \mid U: U \rightarrow \mathbb{R}^{n}$ is an embedding. Let $v_{f}(p)$ denote the $(n-m)$-plane which is normal to $f(U)$ at $f(p)$. Then the total space of the normal bundle is $N_{f}=\left\{(p, x) \in M \times \mathbb{R}^{n}: x \in\right.$ $\left.v_{f}(p)\right\}$. The projection map $\eta_{f}: N_{f} \rightarrow \mathbb{R}^{n}$ is defined by $\eta_{f}(p, x)=x$ and the set of focal points with base $p$ is $\Gamma_{f}(p)=\left\{x \in \mathbb{R}^{n}:(p, x)\right.$ is a singularity of $\left.\eta\right\}$. For each $p \in M, \Gamma_{f}(p)$ is a real algebraic variety in $v_{f}(p)$ which can be defined as the zeros of a polynomial on $v_{f}(p)$ of degree $\leq m$.

DEfinition. The immersion $f: M \rightarrow \mathbb{R}^{n}$ has totally reducible focal set if, for all $p \in M$, $\Gamma_{f}(p)$ can be defined as the zeros of a real polynomial which is a product of real linear factors.

So each irreducible component of $\Gamma_{f}(p)$ is an affine hyperplane in $v_{f}(p)$. There are other ways of describing this property; it is shown in $[7,10]$ that $f$ has totally reducible focal set if and only if $f$ has flat normal bundle, where $M$ is thought of as a Riemannian manifold with metric $g$ induced from $\mathbb{R}^{n}$. An intermediate step, which is hard to extract from the literature, is to show that the property of having totally reducible focal set implies that the shape operators $A_{u}, A_{v}$ commute, for any two normals $u, v$ based at the same point of $M$. A more general version of this result is proved in [4, Lemma 3.2]. It is known that such commuting operators have common eigenspaces. In the present context this means that the curvature eigenspaces depend only on $p \in M$ and not on a choice of normal direction. From this it is straightforward to deduce that $\Gamma_{f}(p)$ is a union of hyperplanes $H_{i}=\left\{x \in v_{f}(p):\left\langle x-f(p), n_{i}\right\rangle=1\right\}, i=1, \ldots, k$, if and only if for each curvature eigenspace $E_{i}$ at $p$ (corresponding to $H_{i}$ ), $i=1, \ldots, k$,

$$
X \in E_{i} \Rightarrow \mathrm{II}(X, Y)=g(X, Y) n_{i} \text { for all } Y \in T_{p} M,
$$

$\dagger$ The second author was supported by Uludag University, Turkey.

Glasgow Math. J. 36 (1994) 11-16. 
where II is the second fundamental form of the immersion $f$. The property that an immersion has totally reducible focal set can then be seen to be a conformal invariant. As was pointed out by the referee this follows from the formula giving the change in the second fundamental form when the immersion is composed with a conformal transformation of $\mathbb{R}^{n}[1$, p. 60]. So, we look for immersions whose images do not lie on hyperspheres or hyperplanes in the ambient space.

An immersion $f: M \rightarrow \mathbb{R}^{n}$ is said to be substantial if $f(M)$ is not contained in any affine hyperplane of $\mathbb{B}^{n}$.

Definition. The immersion $f: M \rightarrow \mathbb{R}^{n}$ is nonspherical if $f$ is substantial and $f(M)$ is not contained in any round hypersphere of $\mathbb{R}^{n}$.

3. Embeddings of surfaces with totally reducible focal set. If $M^{2}$ is a projective plane or a projective plane with handles we showed in [5] how to construct a nonspherical immersion $f: M^{2} \rightarrow \mathbb{R}^{n}$ with totally reducible focal set for any $n \geq 4$. Here we show that for $n \geq 5$ there exists a nonspherical embedding $f: M^{2} \rightarrow \mathbb{R}^{n}$ with totally reducible focal set. We use the following result which was proved in [5].

THEOREM 3.1. Let $f: M^{m} \rightarrow \mathbb{R}^{n}$ be an immersion such that for all $p \in M$ either (i) there is a neighbourhood $U$ of $p$ in $M$ such that $f(U)$ is contained in an affine $(m+1)$-plane in $\mathbb{R}^{n}$ or (ii) there is a neighbourhood $U$ of $p$ in $M$ such that $f(U)$ is contained in a round $(m+1)$-sphere in an affine $(m+2)$-plane in $\mathbb{R}^{n}$. Then $f$ has totally reducible focal set.

Our starting point is the immersion of the projective plane, $\mathbb{P}^{2}$, in $\mathbb{R}^{3}$ known as Boy's surface [2]. Descriptions of this immersion can also be found in [3] and [6].

This immersion $g: \mathbb{P}^{2} \rightarrow \mathbb{R}^{3}$ has the following properties. There is an open Möbius band $\mathbb{M} \subset \mathbb{P}^{2}$ such that all the self-intersections of $g$ lie in $\mathbb{M}$. This Möbius band is shown in Figure 1 where the solid curve is the set of self-intersection points. Let $U_{1}, U_{2}, U_{3}$ be the open subsets of $\mathbb{M}$ as shown in Figures 1 and 2. For $i=1,2,3$ let $p_{i} \in U_{i}$ be as shown in Figures 1 and 3, and let $c_{i}$ be the set of points on the open curve in $U_{i}$ as in Figures 1 and 3. So the closure of $c_{i}$ is $c_{i} \cup\left\{p_{i}\right\}$. Let $d_{1}$ be the set of points on the open curve as shown in Figure 4. So the closure of $d_{1}$ is $d_{1} \cup\left\{p_{2}\right\} \cup\left\{p_{3}\right\}$. Similarly for $d_{2}$ and $d_{3}$ as in Figure 1. The immersion $g: \mathbb{P}^{2} \rightarrow \mathbb{R}^{3}$ is such that for $i=1,2,3, g\left(U_{i}\right) \subset\left\{\left(x_{1}, x_{2}, x_{3}\right) \in\right.$ $\left.\mathbb{R}^{3}: x_{i}=0\right\}$. Further $g\left(p_{1}\right)=g\left(p_{2}\right)=g\left(p_{3}\right)=(0,0,0)$ and this is the only triple point. For $i=1,2,3, g\left(c_{i}\right)=g\left(d_{i}\right)$. Elsewhere $g$ is one-one.

Diagrams showing the image of $g \mid M$ can be found in $[3,6]$. We show that points in a neighbourhood of the self-intersection points can be moved into higher dimensions to give an embedding of $\mathbb{P}^{2}$ into $\mathbb{R}^{5}$ which satisfies the hypotheses of Theorem 3.1.

THeOREм 3.2. There is a nonspherical embedding of the projective plane into $\mathbb{R}^{5}$ with totally reducible focal set.

Proof. Let $g: \mathbb{P}^{2} \rightarrow \mathbb{R}^{3}$ be the immersion described above. For $i=1,2,3$ let $\lambda_{i}: \mathbb{P}^{2} \rightarrow$ $[0,1]$ be a smooth function such that $\lambda_{i}(p) \neq 0$ for all $p \in c_{i} \cup\left\{p_{i}\right\}$ and $\operatorname{supp} \lambda_{i} \subset U_{i}$. Put $U=U_{1} \cup U_{2} \cup U_{3}$. Define a smooth immersion $f: \mathbb{P}^{2} \rightarrow \mathbb{R}^{3} \times \mathbb{R} \times \mathbb{R} \equiv \mathbb{R}^{5}$ by

$$
f(p)= \begin{cases}(g(p), 0,0) & \text { if } p \in \mathbb{P}^{2} \backslash U, \\ \left(g(p), \lambda_{1}(p), 0\right) & \text { if } p \in U_{1}, \\ \left(g(p), 0, \lambda_{2}(p)\right) & \text { if } p \in U_{2}, \\ \left(g(p), \lambda_{3}(p), \lambda_{3}(p)\right) & \text { if } p \in U_{3} .\end{cases}
$$




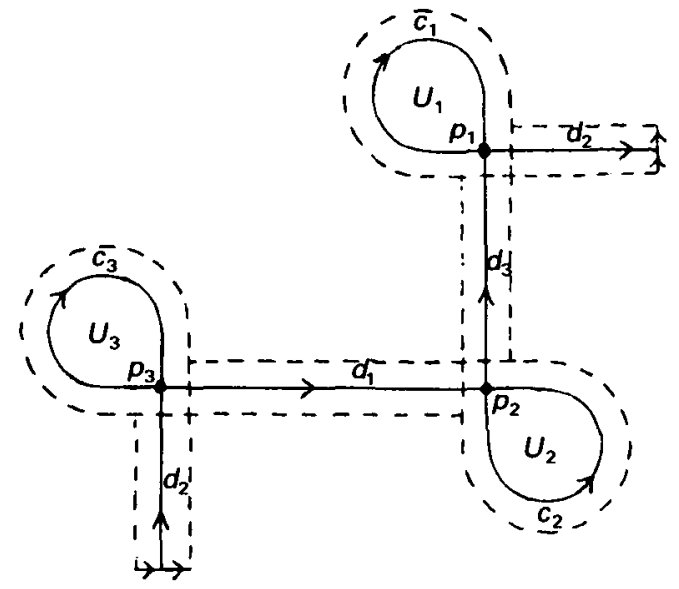

Figure 1

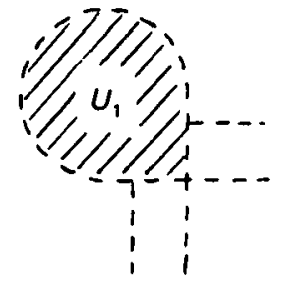

Figure 2

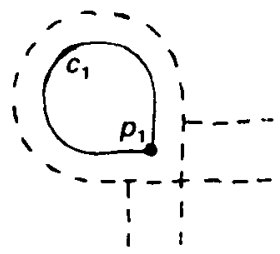

Figure 3

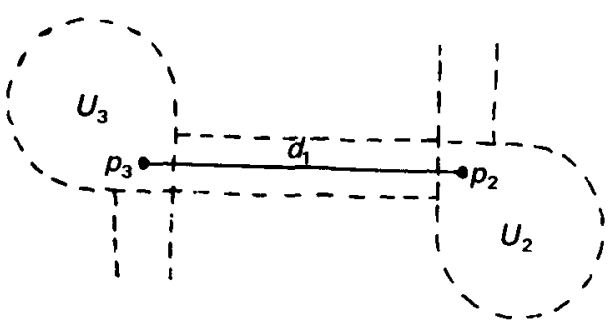

Figure 4

Now $f$ is one-one, for if $f(p)=f(q)$ then $g(p)=g(q)$ and so

(1) $p, q \in\left\{p_{1}, p_{2}, p_{3}\right\}$

or (2) for some $i=1,2,3, p \in c_{i}, q \in d_{i}$ (or vice-versa)

or (3) $p=q$.

Case (1) can only occur if $p=q$, since, for example, if $p=p_{1}$ and $q=p_{3}$, then $f\left(p_{1}\right)=\left(g\left(p_{1}\right), \lambda_{1}\left(p_{1}\right), 0\right) \neq\left(g\left(p_{3}\right), \lambda_{3}\left(p_{3}\right), \lambda_{3}\left(p_{3}\right)\right)=f\left(p_{3}\right)$, as $\lambda_{3}\left(p_{3}\right) \neq 0$.

Case (2) cannot occur, for if $p \in c_{1}$ and $q \in d_{1}$, say, then as $d_{1} \cap U_{1}=\varnothing, f(q)$ is one of $(g(q), 0,0),\left(g(q), 0, \lambda_{2}(q)\right),\left(g(q), \lambda_{3}(q), \lambda_{3}(q)\right)$. So $f(q) \neq f(p)=\left(g(p), \lambda_{1}(p), 0\right)$, as $\lambda_{1}(p) \neq 0$. 
So $f: \mathbb{P}^{2} \rightarrow \mathbb{R}^{5}$ is an embedding. It is clearly substantial since $f \mid\left(\mathbb{P}^{2} \backslash U\right)$ is substantial in $\mathbb{R}^{3} \times\{(0,0)\}$ and $f\left(p_{1}\right) \in \mathbb{R}^{3} \times\left\{\left(\lambda_{1}\left(p_{1}\right), 0\right)\right\}$ with $\lambda_{1}\left(p_{1}\right) \neq 0$ and $f\left(p_{2}\right) \in \mathbb{R}^{3} \times$ $\left\{\left(0, \lambda_{2}\left(p_{2}\right)\right)\right\}$ with $\lambda_{2}\left(p_{2}\right) \neq 0$. Also $f\left(\mathbb{P}^{2} \backslash U\right)$ is not contained in a round 2 -sphere in $\mathbb{R}^{3} \times\{(0,0)\}$. Hence $f$ is nonspherical.

Finally we show that $f$ has totally reducible focal set by observing that for each $p \in \mathbb{P}^{2}$ there is a neighbourhood $V$ of $p$ in $\mathbb{P}^{2}$ such that $f(V)$ is contained in an affine 3-plane in $\mathbb{R}^{5}$.

If $p \in \mathbb{P}^{2} \backslash U$ take $V=\mathbb{P}^{2} \backslash \bigcup\left\{\operatorname{supp} \lambda_{i}: i=1,2,3\right\}$ since $f(V) \subset \mathbb{R}^{3} \times\{(0,0)\}$. For $i=1,2,3$, if $p \in U_{i}$ take $V=U_{i}$ since

$$
\begin{aligned}
& f\left(U_{1}\right) \subset g\left(U_{1}\right) \times \mathbb{R} \times\{0\} \subset\left\{\left(x_{1}, \ldots, x_{5}\right): x_{1}=x_{5}=0\right\}, \\
& f\left(U_{2}\right) \subset g\left(U_{2}\right) \times\{0\} \times \mathbb{R} \subset\left\{\left(x_{1}, \ldots, x_{5}\right): x_{2}=x_{4}=0\right\}
\end{aligned}
$$

and

$$
f\left(U_{3}\right) \subset g\left(U_{3}\right) \times\{(\alpha, \alpha): \alpha \in \mathbb{R}\} \subset\left\{\left(x_{1}, \ldots, x_{5}\right): x_{3}=0, x_{4}=x_{5}\right\} .
$$

Hence by Theorem $3.1 f$ has totally reducible focal set.

COROLlaRy 3.3. Let $M^{2}$ be a projective plane with handles. There is a nonspherical embedding $f: M^{2} \rightarrow \mathbb{R}^{5}$ with totally reducible focal set.

Proof. Modify Boy's immersion of the projective plane in $\mathbb{R}^{3}$ by adding the appropriate number of handles to $\mathbb{P}^{2}$ in $\mathbb{P}^{2} \backslash \mathbb{M}$, where $\mathbb{M}$ is the Möbius band described before Theorem 3.2 , and then use the same construction as in Theorem 3.2.

To get nonspherical immersions with higher codimension we use the following theorem.

THEOREM 3.4. Let $g: M^{m} \rightarrow \mathbb{R}^{k}$ be a nonspherical immersion (resp. embedding) with totally reducible focal set, such that there is an open set $U \subset M$ with $g(U)$ contained in an affine $m$-plane in $\mathbb{R}^{k}$. Then for all $n>k$ there is a nonspherical immersion (resp. embedding ) $f: M^{m} \rightarrow \mathbb{R}^{n}$ with totally reducible focal set.

Proof. For any positive integer $d$, take pairwise disjoint open sets $U_{1}, \ldots, U_{d}$ in $U$ and for $i=1, \ldots, d$ let $\lambda_{i}: M \rightarrow[0,1]$ be a smooth function with $\operatorname{supp} \lambda_{i} \neq \varnothing$ and $\operatorname{supp} \lambda_{i} \subset U_{i}$.

Define $f: M \rightarrow \mathbb{R}^{k} \times \mathbb{R} \times \ldots \times \mathbb{R} \equiv \mathbb{R}^{k+d}$ by

$$
f(p)=\left(g(p), \lambda_{1}(p), \ldots, \lambda_{d}(p)\right) .
$$

Then $f$ is a smooth immersion (resp. embedding). It is nonspherical since $g$ is nonspherical and for each $i \in\{1, \ldots, d\}$ there exists $p \in U_{i}$ with $\lambda_{i}(p) \neq 0$. There is an affine $m$-plane $\Pi$ in $\mathbb{R}^{k}$ such that $g(U) \subset \Pi$. So, for $i=1, \ldots, d$,

$$
f\left(U_{i}\right) \subset\left\{\left(x_{1}, \ldots, x_{k+d}\right):\left(x_{1}, \ldots, x_{k}\right) \in \Pi, x_{k+j}=0 \text { for } j \in\{1, \ldots, d\}, j \neq i\right\} .
$$

Therefore for $p \in U_{i}, f(p) \in f\left(U_{i}\right)$ which is contained in an affine $(m+1)$-plane in $\mathbb{R}^{k+d}$. Hence, as $\Gamma_{f}(p)$ is defined locally, it follows from Theorem 3.1 that $\Gamma_{f}(p)$ is a union of hyperplanes in $v_{f}(p)$. For $p \in M \backslash \bigcup$ supp $\left.\lambda_{i}: i=1, \ldots, d\right\}, f(p)=(g(p), 0, \ldots, 0)$ and the focal set $\Gamma_{g}(p) \equiv \Gamma_{f}(p) \times \mathbb{R}^{d}$ [5, Proposition 2.3]. Hence $f$ has totally reducible focal set. 
COROLlaRy 3.5. Let $h: M^{m} \rightarrow \mathbb{B}^{k}$ be a nonspherical immersion (resp. embedding) with totally reducible focal set such that there is an open set $W \subset M$ and an affine $(m+1)$-plane $\Lambda$ in $\mathbb{R}^{k}$ with $h(W) \subset \Lambda$. Then, for all $n>k$ there is a nonspherical immersion (resp. embedding ) $f: M^{m} \rightarrow \mathbb{R}^{n}$ with totally reducible focal set.

Proof. Modify $h$ to get $g: M \rightarrow \mathbb{R}^{k}$ by flattening part of $h(W)$ so that there is an open set $V \subset W$ with $g(V)$ contained in an affine $m$-plane $\Pi \subset \Lambda$. Then $g$ has totally reducible focal set by the local version of Theorem 3.1. So $g$ satisfies the hypotheses of Theorem 3.4 .

COROLlaRY 3.6. Let $M^{2}$ be a projective plane or a projective plane with handles. Then for any $n \geq 5$ there exists a nonspherical embedding $f: M^{2} \rightarrow \mathbb{R}^{n}$ with toally reducible focal set.

Proof. Let $h: M^{2} \rightarrow \mathbb{R}^{5}$ be a nonspherical embedding as constructed in Theorem 3.2 and Corollary 3.3. If $W=M^{2} \backslash \mathbb{M}$ where $\mathbb{M}$ is the Möbius band described before Theorem 3.2 , then $h(W) \subset \mathbb{B}^{3} \times\{0\} \times\{0\}$. So Corollary 3.5 can be applied to give the required $f: M^{2} \rightarrow \mathbb{R}^{n}, n>5$.

4. Immersions with flat normal bundle. To show that a projective plane or a projective plane with handles cannot be embedded in $\mathbb{R}^{4}$ with totally reducible focal set it is necessary to take a different point of view. As remarked in Section $2, f$ has totally reducible focal set if and only if $f$ has flat normal bundle. The following argument which shows that there does not exist an embedding with flat normal bundle of a projective plane or a projective plane with handles in $\mathbb{R}^{4}$ is based on ideas of $M$. Gromov and $E$. G. Rees.

THEOREM 4.1. Let $M^{2}$ be a projective plane or a projective plane with handles. Then there does not exist an embedding $f: M^{2} \rightarrow \mathbb{R}^{4}$ with totally reducible focal set.

Proof. Suppose there is an embedding $f: M^{2} \rightarrow \mathbb{R}^{4}$ with totally reducible focal set. So $f$ has flat normal bundle. Let $\pi: \hat{M} \rightarrow M$ be a double cover of $M$, so $\hat{M}$ is an orientable surface. Consider $\pi^{*}: H^{2}(M ; \mathscr{Z}) \rightarrow\left(H^{2}(\hat{M} ; \mathbb{Z})\right.$ where $\mathscr{Z}$ denotes the twisted integer coefficient system on $M$. Both groups are infinite cyclic and, in each case, the generator can be taken to be the fundamental class [9]. Since $\pi^{*}$ maps fundamental class to fundamental class it follows that $\pi^{*}$ is an isomorphism. Let $e\left(N_{f}\right) \in H^{2}(M ; \mathscr{Z})$ and $e\left(N_{\hat{f}}\right) \in H(M ; \mathbb{Z})$ denote the Euler classes of the normal bundles of $f$ and $f \circ \pi=\hat{f}$. Then there are integers $\chi\left(N_{f}\right), \chi\left(N_{\hat{f}}\right)$ such that $e\left(N_{f}\right)$ is $\chi\left(N_{f}\right)$ times the generator of $H^{2}(M ; \mathscr{L})$ and $e\left(N_{\hat{f}}\right)$ is $\chi\left(N_{\hat{f}}\right)$ times the generator of $H^{2}(\hat{M} ; \mathbb{Z})$. Now $\hat{f}: \hat{M} \rightarrow \mathbb{R}^{4}$ has flat normal bundle since $f: M \rightarrow \mathbb{R}^{4}$ has flat normal bundle. Therefore, by the Gauss-Bonnet theorem for the normal bundle [8], $\chi\left(N_{\hat{f}}\right)=0$. Now $\chi\left(N_{f}\right)=2 \chi(\bmod 4)$, where $\chi$ denotes the Euler characteristic of $M$ [9], and, as $\chi$ is odd, $\chi\left(N_{f}\right) \neq 0$. But $\pi^{*}\left(e\left(N_{f}\right)\right)=e\left(N_{\hat{f}}\right)$ implies that $\chi\left(N_{f}\right)=\chi\left(N_{\hat{f}}\right)$. This contradiction shows that there does not exist $f: M \rightarrow \mathbb{R}^{4}$ with flat normal bundle.

As observed in Section 2, the property that an immersion has totally reducible focal set is a conformal invariant. Combining this fact with Theorem 4.1 gives the following Corollary.

COROLlaRY 4.2. Let $M^{2}$ be a projective plane or a projective plane with handles. If $f: M^{2} \rightarrow \mathbb{R}^{5}$ has totally reducible focal set then $f$ is nonspherical. 


\section{REFERENCES}

1. A. L. Besse, Einstein manifolds, Springer-Verlag, Berlin, 1987.

2. W. Boy, Über die Curvatura integra und die Topologie geschlossener Flächen, Math. Ann. 57 (1903), 151-184.

3. J. S. Carter, On generalizing Boy's surface: constructing a generator of the third stable stem, Trans. Amer. Math. Soc. 298 (1986), 103-122.

4. S. Carter and A. West, Partial tubes about immersed manifolds, (University of Leeds, Pure Mathematics 1992 Preprint Series, No. 26).

5. S. Carter and R. Ezentas, Immersions with totally reducible focal set, J. Geom. 45 (1992), $1-7$.

6. P. J. Eccles, Multiple points of codimension one immersions, Topology Symposium, Siegen 1979, Lecture Notes in Mathematics 788, pp. 23-28 (Springer-Verlag, Berlin, 1980).

7. A. M. Flegmann, The parallel rank of a submanifold of Euclidean space, Math. Proc. Camb. Phil. Soc. 106 (1989), 89-93.

8. J. A. Little, On singularities of submanifolds of higher dimensional Euclidean spaces. Ann. Mat. Pura Appl. (4) 83 (1969), 261-336.

9. W. S. Massey, Proof of a conjecture of Whitney, Pacific J. Math. 31 (1969), 143-156.

10. R. S. Palais and C. L. Terng, Critical Point Theory and Submanifold Geometry. Lecture Notes in Mathematics 1353 (Springer-Verlag, Berlin, 1988).

School of Mathematics

UNIVERSITY OF LEEDS

LEEDS, LS2 9JT

U.K.
ULUDAG UNIVERSITESI

Fen-Ed. Fak. Mat. Böl.

BURSA

TURKEY 